\title{
Bogoliubov Quasiparticles in Constrained Systems
}

\author{
V.N.Pervushin, V.I.Smirichinski \\ Joint Institute for Nuclear Research, \\ 141980, Dubna, Russia.
}

\begin{abstract}
The paper is devoted to the formulation of quantum field theory for an early universe in General Relativity considered as the Dirac general constrained system. The main idea is the Hamiltonian reduction of the constrained system in terms of measurable quantities of the observational cosmology: the world proper time, cosmic scale factor, and the density of matter. We define " particles" as field variables in the holomorphic representation which diagonalize the measurable density. The Bogoliubov quasiparticles are determined by diagonalization of the equations of motion (but not only of the initial Hamiltonian) to get the set of integrals of motion (or conserved quantum numbers, in quantum theory). This approach is applied to describe particle creation in the models of the early universe where the Hubble parameter goes to infinity.
\end{abstract}




\section{Introduction}

All modern relativistic field theories describe constrained systems with a set of "superfluous" degrees of freedom and pure gauge fields. An identification of physical variables and quantities, in gauge theories and General Relativity (GR), is a long-time problem which stimulated Dirac to formulate, for this aim, the general Hamiltonian theory for constrained systems [1] developed later by many authors (see e.g. monographs [2, 3]). The essence of the Dirac approach to constrained systems is the reduction of the initial extended phase space to separate the true physical variables from the parameters of gauge and general coordinate transformations and to construct a dynamic equivalent unconstrained system in the reduced phase space in terms of gauge-invariant variables which are called the "Dirac observables".

However, the application of the Dirac scheme of the Hamiltonian reduction to field theories invariant with respect to the reparametrization of time (GR and cosmological models) meets with an additional peculiarity: in these theories there are superfluous variables which are excluded by constraints and abandon the sector of physical variables (i.e. "Dirac observables"), but not the sector of measurable quantities [ [4, 5, 6].

Examples of such variables are the cosmic scale factor and the invariant proper time treated as measurable quantities in cosmological models. In this case, set of equations of the reduced theory does not reflect all the physical content of the initial extended theory and must be supplemented by two equations for the superfluous variable and its momentum [5]. The second equation leads to the Friedmann-Hubble law of evolution of the universe (i.e. the dependence of the red shift of spectral lines of the cosmic object atoms on a distance of this object from the Earth); while the first one determines the evolution of measurable density of matter [6].

In the present paper, we would like to reconsider the problem of "particles" and "quasiparticles" in the early universe [8, 9, 10, 11] in the context of the Dirac approach to constrained systems supplemented by the sector of measurable quantities $₫, 0$, 6. 6 .

The basic idea is the definition of "particles" as field variables in the holomorphic representation [2] which diagonalize the measurable density in the supplemented equations of the evolution of the universe.

To get the set of integrals of motion (or conserved quantum numbers in quantum theory), we apply the Bogoliubov transformations of particle variables for diagonalization of the equations of motion (but not only of the initial Hamiltonian [8, 9, 10, 11]), in the correspondence with the original idea in Bogoliubov's paper [12] where these transformations were considered as an effective mathematical tool to construct the energy spectrum of a weakly non-ideal Bose gas.

The Bogoliubov quasiparticles are determined as field variables with conserved "numbers of quasiparticles".

In the next section, we formulate the problem and the model. In Section 3, measurable particles are defined. In Section 4, we introduce the Bogoliubov transformations to diagonalize equations of motion. Section 5 is devoted to the description of creation of massive particles and gravitons in the early universe.

\footnotetext{
${ }^{1}$ It is worth to recall that yet in 1873 J.C.Maxwell wrote [7] "The most important aspect of any phenomenon from mathematical point of view is that of a measurable quantity. I shall therefore consider electrical phenomena chiefly with a view to their measurement describing the methods of measurement and defining the standards on which they depend."
} 


\section{Statement of Problem}

General Relativity

$$
W^{g r}(g)=\int d^{4} x\left[-\sqrt{-g} \frac{\mu^{2}}{6} R(g)+\mathcal{L}_{\text {matter }}\right] \quad\left(\mu^{2}=M_{\text {Planck }}^{2} \frac{3}{8 \pi}\right)
$$

is considered as a constrained system for the Dirac-ADM $3+1$ foliation of the four-dimensional manifold [13

$$
(d s)_{e}^{2}=g_{\mu \nu} d x^{\mu} d x^{\nu}=N^{2} d t^{2}-{ }^{(3)} g_{i j} \breve{d x}^{i} \breve{d x}^{j} ; \quad\left(\breve{d x}^{i}=d x^{i}+N^{i} d t\right)
$$

The matter Lagrangian in the action describes a set of boson and fermion fields. The problems of initial data and classical and quantum Hamiltonian dynamics of these fields in GR are conventionally formulated in terms of the so-called Lichnerowicz conformal-invariant field variables and metric 17, 18

$$
{ }^{(n)} f_{c}(x)={ }^{(n)} f(x)\left\|^{(3)} g(t, x)\right\|^{-n / 6} ; \quad(d s)_{c}^{2}=N_{c}^{2} d t^{2}-{ }^{(3)} g_{(c) i j} \breve{d x^{i}} \breve{d x^{j}} ; \quad\left\|{ }^{(3)} g_{c}\right\|=1,
$$

where $(n=-1,-3 / 2,0,2)$ is the conformal weight for scalar, vector, spinor, and tensor fields, respectively, $N_{c}$ is the conformal lapse function, $d s_{c}$ is the conformal interval.

As a result, the action of GR in terms of the conformal variables (3) has the structure [5] of the Penrose-Chernikov-Tagirov action [19 for the conformal scalar field

$$
W_{\text {tot }}^{g r}\left(\varphi_{g}, f_{c}\right)=\int d t d^{3} x\left[-N_{c} \frac{\varphi_{g}^{2}}{6}{ }^{(4)} R_{c}+\varphi_{g} \partial_{\mu}\left(N_{c} \partial^{\mu} \varphi_{g}\right)+N_{c}\left(\mathcal{L}_{\left(\varphi_{g}=0\right)}-\varphi_{g} F+\varphi_{g}^{2} B\right)\right],
$$

where $\mathcal{L}_{\left(\varphi_{g}=0\right)}$ is the massless part of the matter Lagrangian, $B$ and $F$ are the mass terms of the boson and fermion fields, respectively, both the Newton constant and masses of elementary particles are formed by the metric scale field $\varphi_{g}$

$$
\varphi_{g}=\mu\|\|^{(3)} g \|^{1 / 6} .
$$

Action (4) can be considered also as scalar version [5, 6] of the Weyl conformal theory [20], where the metric scale field (5) plays the role of the Lichnerowicz variable (3) of a scalar field considered as the measure of a change of the length of a vector in its parallel transport and, simultaneously, as a modulus of the Higgs scalar field in the Standard Model of unification of electroweak and strong interactions. Dynamics of both the theories (the Einstein GR and the Weyl one) is the same, but not standards of measurement. An Einstein observer measures the absolute lengths $(d s)_{e}$, while a Weyl observer can measure only the ratio of lengths of two vectors $(d s)_{w}=\left(d s_{1}\right)_{e} /\left(d s_{2}\right)_{e}=\left(d s_{1}\right)_{c} /\left(d s_{2}\right)_{c}$ which is conformal-invariant.

The Dirac-ADM-action and interval (2) are invariant with respect to transformations of a kinemetric subgroup of the group of general coordinate transformations [14, 5, 15]

$$
t \rightarrow t^{\prime}=t^{\prime}(t) ; \quad x_{i} \rightarrow x_{i}^{\prime}=x_{t}^{\prime}\left(t, x_{1}, x_{2}, x_{3}\right),
$$

This group of diffeomorphism of the Hamiltonian description of GR includes reparametrizations of the coordinate time which could not be treated as the "observable" evolution parameter of the reparametrization-invariant Hamiltonian dynamics of the universe.

One of the main problems of the Hamiltonian reduction in GR is to pick out the global variable which can represent the internal evolution parameter of the corresponding reduced system. 
There is a lot of speculations on this subject, here we try to continue these attempts in the spirit of identification of this "internal evolution parameter" in GR with the global component of the metric scale field (5) (or, the scalar field in the Weyl theory [6]) [18, 5, 6]

$$
\varphi_{g}(t, x)=\mu\left\|^{(3)} g(t, x)\right\|^{1 / 6}=\varphi_{0}(T) a(T, x) .
$$

Here $T$ is the world conformal time formed by the global component $N_{0}$ of the conformal lapse function:

$$
N_{c}(t, x)=N_{0}(t) \mathcal{N}(T, x) ; \quad d T=N_{0}(t) d t .
$$

Fields $\mathcal{N}(T, x) ; \quad a(T, x)$ begin from unit, $\mathcal{N}(T, x)=1+\ldots$, in the cosmological perturbation theory [16] and form interactions of the fields with the positive contribution to the energy constraint.

When interactions are neglected $\left(a=\mathcal{N}=1 ; \quad N^{k}=0 ; g_{i j}^{c}=\delta_{i j}+h_{i j}\right)$, the GR action is reduced to the action of the well-known system of "free" conformal fields in a finite space-volume $\left(V=\int d^{3} x\right)$ [8, 9, 10, 11]

$$
W^{E}\left[P_{f}, f ; P_{0}, \varphi_{0} \mid t\right]=\int_{t_{1}}^{t_{2}} d t\left(\left[\int d^{3} x \sum_{f} P_{f} \dot{f}\right]-\dot{\varphi}_{0} P_{0}-N_{0}\left[-\frac{P_{0}^{2}}{4 V}+H_{0}\right]+\frac{1}{2} \partial_{0}\left(P_{0} \varphi_{0}\right)\right),
$$

where $H_{0}$ is a sum of the Hamiltonians of "free" fields, graviton (h), photon (p), massive vector $(\mathrm{v})$, and spinor $(\mathrm{s}))$

$$
\begin{gathered}
H_{0}=H_{M}+H_{R}+H_{h} \\
H_{M}=\frac{1}{2} \int d^{3} x\left(P_{(v)}^{2}+\left(\partial_{i} v\right)^{2}+\left(y_{b} \varphi_{0}\right)^{2} v^{2}\right)+\int d^{3} x \bar{\psi}\left(y_{s} \varphi_{0}-i \gamma_{j} \partial_{j}\right) \psi,
\end{gathered}
$$

is the Hamiltonian of massive conformal fields, where the role of masses is played by the "internal evolution parameter" $\varphi_{0}$ multiplied by dimensionless constants $y, H_{R}$ is the Hamiltonian of massless fields with $y=0$, and $H_{h}$ is the Hamiltonian of gravitons

$$
H_{h}=\int d^{3} x\left(\frac{6\left(P_{(h)}^{T}\right)^{2}}{\varphi_{0}^{2}}+\frac{\varphi_{0}^{2}}{24}\left(\partial_{i} h^{T}\right)^{2}\right) ; \quad\left(h_{i i}^{T}=0 ; \quad \partial_{j} h_{j i}^{T}=0\right),
$$

(where the last two equations follow from the separation of the determinant of three-dimensional metric (3) and from the momentum constraint). There is a direct correspondence between a particle in Special Relativity (SR)

$$
W^{E}=\int d \tau\left[-p_{\mu} \dot{x}^{\mu}-\frac{N}{2 m}\left(-p_{\mu}^{2}+m^{2}\right)\right] ; \quad \mu=(0,1,2,3)
$$

and the universe in GR: for the unobservable coordinate times $(\tau \rightarrow t)$; the internal evolution parameter $\left(x_{0} \rightarrow \varphi_{0}\right)$; the reduced phase space variables $\left(x_{i}, p_{i} \rightarrow P_{f}, f\right) ; \quad$ and the proper time $\quad\left(d T=N d \tau \rightarrow d T=N_{0} d t\right)$. Recall that the conformal world time $T$ is considered in the Weyl theory as the time measured by an observer in the comoving frame, while in the Einstein GR, an observer measures in the comoving frame the Friedmann world time $\left(T_{f}\right)$ connected with $T$ by the relation

$$
\mu d T_{f}(T)=\varphi_{0}(T) d T \text {. }
$$

Finally, we get the following statement of the problems considered in the present paper:

to fulfil the Hamiltonian reduction in the theory described by action (9), and to quantize the reduced version of the theory. 


\section{Evolution of the universe and "particles"}

The Hamiltonian reduction of the system described by action (9) means explicit solving of the constraint

$$
\frac{\delta W_{0}^{E}}{\delta N_{0}}=0 \Rightarrow H=\left[-\frac{P_{0}^{2}}{4 V}+H_{0}\right]=0 \Rightarrow\left(P_{0}\right)_{ \pm}= \pm 2 \sqrt{V H_{0}} \equiv \pm H^{R} .
$$

This equation has two solutions which correspond to two reduced systems with the actions

$$
W_{ \pm}^{R}\left(P_{f}, f \mid \varphi_{0}\right)=\int_{\varphi_{1}=\varphi_{0}\left(t_{1}\right)}^{\varphi_{2}=\varphi_{0}\left(t_{2}\right)} d \varphi_{0}\left\{\left(\int d^{3} x \sum_{f=h^{T}, p, v, s} P_{f} \partial_{\varphi_{o}} f\right) \mp H^{R} \pm \frac{1}{2} \partial_{\varphi_{o}}\left(H^{R} \varphi_{0}\right)\right\}
$$

where the scale factor $\varphi_{0}$ plays the role of the evolution parameter and $H^{R}$ is the corresponding Hamiltonian of evolution.

The local equations of motion of systems (16) reproduce the invariant sector of the initial extended system and determine the evolution of fields $\left(P_{f}, f\right)$ with respect to the internal evolution parameter $\varphi_{0}$

$$
P_{f}(x, t), f(x, t) \rightarrow P_{f}\left(x, \varphi_{0}\right), f\left(x, \varphi_{0}\right) .
$$

The reduced quantum system is described by the Schrödinger type equation

$$
\frac{d}{i d \varphi_{0}} \Psi_{R}\left(\varphi_{0} \mid f\right)= \pm H^{R} \Psi_{R}\left(\varphi_{0} \mid f\right)
$$

which is a square root of the Wheeler-DeWitt equation $\hat{H} \Psi_{w d w}=0$.

We should emphasize that no one reduced scheme (the classical one and two quantum) contains the "evolution of the universe" in terms of the world time $T$ measured by an observer in the comoving frame, as no one reduced scheme contains this time $d T=N_{0} d t$.

The "evolution of the universe" is the dependence of the world time $T$ on the internal evolution parameter $\varphi_{0}$. This dependence is described by the equation for the "superfluous" momentum $P_{0}$ (which is omitted by the reduced action $W^{R}$ )

$$
\frac{\delta W^{E}}{\delta P_{0}}=0 \Rightarrow\left(\frac{d \varphi_{0}}{N_{0} d t}\right)_{ \pm}=\frac{\left(P_{0}\right)_{ \pm}}{2 V}= \pm \sqrt{\rho\left(\varphi_{0}\right)} ; \quad \rho=\frac{H_{0}}{V}
$$

A solution of the latter

$$
T\left(\varphi_{0}\right)=\int_{0}^{\varphi_{0}} d \varphi \rho^{-1 / 2}(\varphi)
$$

is treated in cosmology as the theoretical description of the Hubble red shift of spectral lines of atoms on cosmic objects at a distance $D$ from the Earth observer:

$$
Z(D)=\frac{\varphi_{0}\left(T_{f}\right)}{\varphi_{0}\left(T_{f}-D / c\right)}-1=\mathcal{H}_{H u b}^{f}\left(T_{f}\right) D / c+\ldots ; \quad \mathcal{H}_{H u b}^{f}\left(T_{f}\right)=\frac{d \varphi_{0}\left(T_{f}\right)}{\varphi_{0}\left(T_{f}\right) d T_{f}}
$$

(where $\mathcal{H}_{H u b}^{f}\left(T_{f}\right)$ is the Hubble parameter).

The redshift-data $Z(D)$ determine the observational dependence of the world proper time on the internal evolution parameter (here, the cosmic scale factor $\varphi_{0}$ ). 
For a relativistic particle described by action (13) the dependence of the proper time $T$ (measured by an observer in the comoving frame) with respect to the internal evolution parameter $\left(x_{0}\right)$ (measured by another observer in the rest frame) is nothing but the dynamic version of the Lorentz transformation.

There is an essential physical difference between Special Relativity (SR) and General Relativity (GR). In SR, two times (the proper time $T$ and internal evolution parameter $x_{0}$ ) are measured in two different frames (the comoving and rest ones) which exclude each other. While in GR, both the times, $T$ and $\varphi_{0}$, are measured by an observer in the comoving frame. The relation between these two times forms the evolution of the universe (19). This evolution depends on standards of measurement of the time.

The same GR dynamics (with the same Wheeler-DeWitt wave function) corresponds to different cosmological pictures for different observers: an Einstein observer, who supposes that he measures an absolute interval, obtains the Friedmann-Robertson-Walker (FRW) cosmology where the red shift is treated as expansion of the universe; a Weyl observer, who supposes that he measures a relative interval $D_{c}$, obtains the Hoyle-Narlikar cosmology [21]. The red shift and the Hubble law in the Hoyle-Narlikar cosmology [21]

$$
Z\left(D_{c}\right)=\frac{\varphi_{0}(T)}{\varphi_{0}\left(T-D_{c} / c\right)}-1 \simeq \mathcal{H}_{H u b}^{c} D_{c} / c ; \quad \mathcal{H}_{H u b}^{c}=\frac{1}{\varphi_{0}(T)} \frac{d \varphi_{0}(T)}{d T}=\frac{\sqrt{\rho(T)}}{\varphi_{0}(T)}
$$

reflect the change of the size of atoms in the process of evolution of masses [21, [1].

Equation (21) give the relation between the present-day value of the scalar field and the cosmological observations (the density of matter and the Hubble parameter)

$$
\varphi_{0}(T)=\frac{\sqrt{\rho_{0}(T)}}{\mathcal{H}_{\text {Hub }}^{c}(T)} .
$$

This value coincides with the Newton constant (or the Planck mass) in limits of the observational data

$$
\frac{\varphi_{0}^{2}\left(T=T_{0}\right)}{6}=\frac{\mu^{2} \Omega_{0}}{6}=\frac{M_{P l}^{2} \Omega_{0}}{16 \pi} \Rightarrow \varphi_{0}\left(T_{0}\right)=\mu \Omega_{0}^{1 / 2} .
$$

The present-day mean matter density

$$
\rho_{b}=\Omega_{0} \rho_{c r} ; \quad\left(\rho_{c r}=\frac{3 \mathcal{H}_{H u b}^{c}}{8 \pi} M_{P l}^{2}\right)
$$

is estimated from observational data on luminous matter $\left(\Omega_{0}=0.01\right)$, the flat rotation curves of spiral galaxies $\left(\Omega_{0}=0.1\right)$, and other data [22] $\left(0.1<\Omega_{0}<2\right)$.

The comparison of direct observations of masses and numbers of particles in the universe with the density of matter measured by the Hubble law (20), or (21), in the context of quantum field theory, means the particle-like treatment of the corresponding Hamiltonian for free fields $H_{0}$ which forms this density. The particle-like representation of $H_{0}$ has the form

$$
H_{0}=\sum_{k ; f=h, p, v, s} \omega_{a_{f}}\left(k ; \varphi_{0}\right) \hat{\mathcal{N}}_{a_{f}}
$$

where $\hat{\mathcal{N}}_{a_{f}}$ is the "number of particles"

$$
\left.\hat{\mathcal{N}}_{a_{f}}=\left\{a_{f}^{+} a_{f}\right\}_{ \pm}=\frac{1}{2}\left(a_{f}^{+} a_{f} \pm a_{f} a_{f}^{+}\right)\right) .
$$


$\left((+)\right.$ corresponds to bosons; and $(-)$, to fermions); and $\omega_{a_{f}}\left(k ; \varphi_{0}\right)$ is the one-particle energy

$$
\omega_{a_{f}}\left(k ; \varphi_{0}\right)=\sqrt{k^{2}+\left(y_{f} \varphi_{0}\right)^{2}}
$$

for gravitons (h) and photons (p) with $(y=0)$, and for bosons $(\mathrm{v})$ and fermions $(\mathrm{s})$ with $(y \neq 0)$.

Thus, the concepts of measurable times lead to concepts of the measurable Hamiltonian and "particles". In contrast with the conventional approach [8, 9, 10, 11] (where "particles" are defined as constant coefficients of the decomposition of field variables over classical solutions), we define the "observable particles" as field dynamic variables in the holomorphic representation which diagonalize the observable Hamiltonian $H_{0}$ in the form (25).

This holomorphic representation of field variables [2] is

$$
\begin{gathered}
f=\sum_{(k, \alpha)} \frac{\exp (i k x)}{V_{0}^{3 / 2} \sqrt{2 \omega_{a_{f}}\left(k, \varphi_{0}\right)}}\left(a_{f}^{+}(-k, \alpha \mid t) \epsilon(-k, \alpha)+a_{f}(k, \alpha \mid t) \epsilon(k, \alpha)\right), \\
P_{f}=i \sum_{(k, \alpha)} \frac{\exp (i k x) \sqrt{\omega_{a_{f}}\left(k, \varphi_{0}\right)}}{V_{0}^{3 / 2} \sqrt{2}}\left(a_{f}^{+}(-k, \alpha \mid t) \epsilon(-k, \alpha)-a_{f}(k, \alpha \mid t) \epsilon(k, \alpha)\right)
\end{gathered}
$$

with operators of creation - $a^{+}$and annihilation - $a$ of particles with the momentum $k$, polarization $\epsilon(k, \alpha)$, and energy $\omega_{a_{f}}\left(k, \varphi_{0}\right)$, and $f_{h}=h^{T} \sqrt{12 \varphi_{0}} ; \quad P_{h}=P_{h}^{T} / \sqrt{12 \varphi_{0}}$.

In terms of the "particle" variables the extended action (9) has the form

$$
W_{0}^{E}=\int_{t_{1}}^{t_{2}} d t\left[-P_{0} \dot{\varphi}_{0}+N_{0} \frac{P_{0}^{2}}{4 V}-\sum_{f, k \alpha}\left(\frac{i}{2} \bar{\chi}_{a_{f}}(k, t) \partial_{t} \chi_{a_{f}}(k, t)-N_{0} \frac{1}{2} \bar{\chi}_{a_{f}}(k, t) \hat{H}_{a_{f}}(k, t) \chi_{a_{f}}(k, t)\right)\right],
$$

here

$$
\bar{\chi}_{a_{f}}=\left(a_{f},-a_{f}^{+}\right) ; \quad \chi_{a_{f}}=\left(\begin{array}{c}
a_{f}^{+} \\
a_{f}
\end{array}\right) ; \quad \hat{H}_{a_{f}}=\left|\begin{array}{ccc}
\omega_{a_{f}} & , & -i \Delta_{f} \\
-i \Delta_{f} & , & -\omega_{a_{f}}
\end{array}\right| .
$$

This action includes additional nondiagonal terms describing the creation of matter in the evolution of the universe:

$$
\Delta_{p}=0 ; \quad \Delta_{v}=\frac{d}{d T} \log \sqrt{\frac{\omega_{v}(k, T)}{\omega_{v}(k, 0)}} ; \quad \Delta_{s}=\frac{d \varphi}{d T}<\frac{k_{i} \gamma_{i} \gamma_{0} y_{s}}{2 \omega_{s}(k, T)^{2}}>; \quad \Delta_{h}=2 \frac{d}{d T} \log \left(\frac{\varphi(T)}{\varphi(0)}\right)
$$

where $<>$ denotes the matrix element between states with definite spins, and $\omega(k, 0), \varphi(0)$ is defined by initial data. In the following, for simplicity, we consider photons $\left(\Delta_{p}=0\right)$, massive bosons $(v)$, and gravitons $(h)$.

On the present-day stage, $\Delta_{f} \sim 0$ and numbers of all particles are conserved. In this case, the proper time dynamics with a fixed number of massive particles $(k=0)$ leads to the Hubble law for the dust stage of classical universe and the corresponding wave function of the universe expressed in terms of the proper time has the form 四

$$
\Psi_{R}\left(\varphi_{0}(T) \mid f\right)=\exp \left(i M_{d u s t} T_{f}\right)<f \mid M_{\text {dust }}>; \quad\left(\mu d T_{f}=\varphi_{0}(T) d T\right),
$$

where $M_{\text {dust }}$ is a sum of all particle masses of the universe, $\langle f \mid M\rangle$ is the product of oscillator wave functions, $T_{f}$ is the Friedmann time and $T$ is the conformal one. As we have seen above, 
the quantum universe filled in by a fixed number $\left(10^{88}\right)$ of massless particles (photons) is described by the wave function

$$
\Psi_{R}\left(\varphi_{0}(T) \mid f\right)=\exp \left(i E_{\text {radiation }} T\right)<f \mid E_{\text {radiation }}>
$$

and by the Hubble law of the classical universe in the radiation stage [四]. Thus, the separation of the global observable time and energies allows us to unify classical and quantum field theory with the classical and quantum cosmologies and to give the perspective of the construction of quantum gravity as a quantum version of the cosmological perturbation theory [16].

In the early universe, $\Delta \neq 0$ and numbers of particles are not conserved

$$
\frac{d \hat{\mathcal{N}}_{f}(T)}{d T} \neq 0
$$

The problem is to find integrals of motion of time-reparametrization system (29) and to describe creation of "particles" in the evolution of the universe.

\section{Bogoliubov quasiparticles: integrals of motion}

To construct integrals of motion in the context of the above-mentioned Hamiltonian reduction, we use the Bogoliubov transformations [12] of "particle" variables

$$
b^{+}=\alpha^{*} a^{+}+\beta^{*} a, \quad b=\alpha a+\beta a^{+} ; \quad|\alpha|^{2}-|\beta|^{2}=1,
$$

which diagonalize the corresponding classical equations expressed in terms of "particles" $\left(a^{+}, a\right)$, so that the "number of quasiparticles" is conserved

$$
\frac{d \mathcal{N}_{b}(t)}{d t} \equiv \frac{d\left(b^{+} b\right)}{d t}=0
$$

The classical equations for action (29) can be written as

$$
i \frac{d}{d T} \chi_{a}=-\hat{H}_{a} \chi_{a}
$$

After Bogoliubov transformations (32)

$$
\chi_{b}=\hat{O} \chi_{a} ; \quad \hat{O}=\left(\begin{array}{cc}
\alpha^{*}, & \beta^{*} \\
\beta, & \alpha
\end{array}\right) ; \quad \hat{O}^{-1}=\left(\begin{array}{rr}
\alpha, & -\beta^{*} \\
-\beta, & \alpha^{*}
\end{array}\right) \text {; }
$$

this equation gets the form

$$
i \frac{d}{d T} \chi_{b}=\left[-i \hat{O}^{-1} \frac{d}{d T} \hat{O}-\hat{O}^{-1} \hat{H}_{a} \hat{O}\right] \chi_{b} \equiv-\hat{H}_{b} \chi_{b} .
$$

Let us require $\hat{H}_{b}$ to be diagonal

$$
\hat{H}_{b}=\left(\begin{array}{cc}
\omega_{b}, & 0 \\
0, & -\omega_{b}
\end{array}\right) .
$$


This means that $\alpha$ and $\beta$ satisfy the equations

$$
\begin{gathered}
\omega_{b}=\left(|\alpha|^{2}+|\beta|^{2}\right) \omega_{a}-i\left(\beta^{*} \alpha-\beta \alpha^{*}\right) \Delta-i\left(\beta^{*} \partial_{T} \beta-\alpha \partial_{T} \alpha^{*}\right), \\
0=2 \beta \alpha \omega_{a}-i\left(\alpha^{2}-\beta^{2}\right) \Delta-i\left(\alpha \partial_{T} \beta-\beta \partial_{T} \alpha\right) .
\end{gathered}
$$

For

$$
\alpha=\cosh (r) e^{i \theta} ; \quad \beta=i \sinh (r) e^{-i \theta}
$$

these equations convert into

$$
\begin{aligned}
\omega_{b} & =\omega_{a} \cosh 2 r-\Delta \sinh 2 r \cos 2 \theta-\cosh 2 r \partial_{T} \theta \\
0 & =\omega_{a} \sinh 2 r-\Delta \cosh 2 r \cos 2 \theta-\sinh 2 r \partial_{T} \theta \\
0 & =\Delta \sin 2 \theta+\partial_{T} r
\end{aligned}
$$

If we consider these equations for constant $\omega_{a}, \Delta$, we get the result of Bogoliubov paper 12

$$
\theta=0 ; \cosh 2 r=\frac{\omega_{a}}{\omega_{b}} \quad \omega_{b}=\sqrt{\omega_{a}^{2}-\Delta^{2}}
$$

Finally , the classical equations in terms of "quasiparticles" are of the form

$$
\frac{d}{d T} b^{+}=i \omega_{b} b^{+} ; \quad \frac{d}{d T} b=-i \omega_{b} b
$$

with the solution

$$
b^{+}=\exp (i Q) b_{0}^{+} ; \quad b=\exp (-i Q) b_{0} ; \quad Q=\int^{T} d T^{\prime} \omega_{b}\left(T^{\prime}\right)
$$

and the conserved number of quasiparticles

$$
\hat{\mathcal{N}}_{b}(t)=\left\{b^{+}(t) b(t)\right\}=\left\{b_{0}^{+} b_{0}\right\}
$$

where $b_{0}^{+}$and $b_{0}$ are initial data.

To close equation (43), we recall that the evolution of the universe is determined by the density of "observable particles"

$$
\begin{aligned}
& \frac{d \varphi}{d T}=\sqrt{\rho(\varphi)} ; \quad \rho(\varphi)=\frac{H_{0}}{V}=\frac{\sum_{f} \omega_{f}(\varphi)\left\{a_{f}^{+} a_{f}\right\}}{V_{0}} ; \\
& \left\{a^{+} a\right\}=\left\{b^{+} b\right\} \cosh 2 r-\frac{i}{2}\left(b^{+2}-b^{2}\right) \sinh 2 r
\end{aligned}
$$

Equations (41)-(47) represent a complete set of equations of classical theory in terms of the holomorphic variables. This theory has a set of integrals of motion (45) which should be converted into the quantum numbers of the corresponding quantum reduced system in agreement with the correspondence principle. 


\section{Creation of Particles}

As $\varphi_{0}$ left the phase space to convert into the internal evolution parameter, in quantum theory we can quantize only the particle sector

$$
\left[a, a^{+}\right]=1 ; \quad\left[b, b^{+}\right]=1 .
$$

In the following, we restrict ourselves to the universe in the state of vacuum of quasiparticles (i.e. the squeezed vacuum):

$$
{ }_{b}<0\left|b^{+} b\right| 0>{ }_{b}=0 ;<0\left|\left\{a^{+} a\right\}\right| 0>=\frac{1}{2} \cosh 2 r=\frac{1}{2} \mathcal{N}_{0}\left(\varphi_{0}\right) .
$$

The quantum equation for the early universe coincides with the Schrödinger one, and the wave function of the state of "nothing" is defined as the squeezed vacuum $b_{f}(k, 0) \mid 0>{ }_{b}=0$ with the scale factor and the particle numbers given by eqs. (46) and (47).

We can rewrite eqs. (29) in terms of the number of particles $\mathcal{N}_{0}(\varphi)$

$$
\begin{aligned}
& \omega_{b_{f}}=\omega_{f} \mathcal{N}_{0}-\Delta_{f}\left[\sqrt{\mathcal{N}_{0}^{2}-1} \cos 2 \theta+\mathcal{N}_{0} 2 \frac{d \theta}{d \tau_{f}}\right] \\
& \omega_{f} \sqrt{\mathcal{N}_{0}^{2}-1}=\Delta_{f}\left[\mathcal{N}_{0} \cos 2 \theta+\sqrt{\mathcal{N}_{0}^{2}-1} 2 \frac{d \theta}{d \tau_{f}}\right] \\
& \sqrt{\mathcal{N}_{0}^{2}-1} \sin 2 \theta=-\frac{d \mathcal{N}_{0}}{d \tau_{f}} ; \quad \Delta_{f}=\sqrt{\rho} \frac{d \tau_{f}\left(\varphi_{0}\right)}{2 d \varphi_{0}}
\end{aligned}
$$

with

$$
\tau_{h}\left(\varphi_{0}\right)=2 \log \left(\frac{\varphi_{0}(T)}{\varphi_{0}(0)}\right) ; \quad \omega_{h}=\sqrt{k^{2}} ; \quad \tau_{v}\left(\varphi_{0}\right)=\log \left(\frac{\omega_{v}\left(\varphi_{0}\right)}{\omega_{0}}\right) ; \quad \omega_{v}\left(\varphi_{0}\right)=\sqrt{k^{2}+\left(y_{v} \varphi_{0}\right)^{2}}
$$

where $\omega_{0}, \varphi_{0}(0)$ are the initial data. ¿From first two equations (50) we get

$$
\omega_{b_{f}}=\frac{\Delta_{f} \cos 2 \theta}{\sqrt{\mathcal{N}_{0}^{2}-1}}
$$

There are two different regimes $\omega_{a_{f}} \gg \Delta_{f},\left(\Delta_{f}=0\right)$ and $\omega_{a_{f}} \ll \Delta_{f}$, for which eqs. (50) can be solved exactly.

For $\Delta_{f}=0$ we got $\theta=0, \mathcal{N}_{0}=1$, particles are not created.

In the opposite case, for $\Delta \rightarrow \infty$, the solution to these equations has the functional form

$$
\theta_{f}=\frac{\pi}{4} ; \quad \mathcal{N}_{0}\left(\varphi_{0}\right)=\frac{1}{2}\left(\frac{\omega_{0}}{\omega_{a}\left(\varphi_{0}\right)}+\frac{\omega_{a}\left(\varphi_{0}\right)}{\omega_{0}}\right)
$$

for massive particles $(f=v)$, and

$$
\theta_{f}=\frac{\pi}{4} ; \quad \mathcal{N}_{0}\left(\varphi_{0}\right)=\frac{1}{2}\left(\frac{\varphi_{0}^{2}(0)}{\varphi_{0}^{2}(T)}+\frac{\varphi_{0}^{2}(T)}{\varphi_{0}^{2}(0)}\right)
$$

for gravitons $(f=h)$. In both the cases $(v, h)$, this solution has the zero energy of the Bogoliubov quasiparticles $\left(\omega_{b_{f}}=0\right)$. In this approximation, the density of gravitons

$$
<\rho(\varphi)>=\rho_{0} \frac{\mathcal{N}_{0}(\varphi)}{2}
$$


(where $\rho_{0}$ is the vacuum density) corresponds to the Friedmann cosmological model with the "rigid" state in the three-dimensional space with a negative curvature.

The proper time dynamics is determined by the integral

$$
T\left(\varphi_{0}\right)=\int_{0}^{\varphi_{0}} d \varphi \frac{1}{\sqrt{<\rho(\varphi)>}}
$$

which leads to the following redshift-formula of the universe evolution

$$
\frac{\varphi_{0}^{2}(T)}{\varphi_{0}^{2}(0)}=\sinh \left(\frac{2 T}{T_{0}}\right) ; \quad T_{0}=\varphi_{0} \sqrt{\frac{2}{\rho_{0}}} .
$$

We can see that for a Weyl observer (with the relative standard of length) there can be a period of the very fast inflation-like evolution of the cosmic scale factor, while an Einstein-Friedmann observer (with the absolute standard of the length) sees the linear dependence of the measured proper time on the scale factor. Thus, ten billion years for an observer with the absolute standard can convert into the "biblical" short period of several thousand years for an observer with the relative standard.

\section{Conclusion}

We have considered the concepts of "particle" and "quasiparticle" in the field theory with the time-reparametrization invariance and the energy constraint, using as an example the description of the universe in General Relativity.

The peculiarity of constrained systems is the presence of superfluous degrees of freedom. Removing these superfluous degrees of freedom from the constrained theory is the aim of the Hamiltonian reduction which has to represent (according to the Dirac theory) an equivalent unconstrained system limited by the "sector of the Dirac observables".

However, in the considered case the "sector of the Dirac observables" does not include all "measurable quantities". The superfluous variables, leaving the phase space, remain in the theory as measurable quantities out of the "sector of the Dirac observables" of the reduced version. In particular, to describe in the generalized Hamiltonian theory the Hubble law of evolution of the universe (measured in observational cosmology) we are enforced to remain in the theory two time-like variables: the world proper time (measured in the comoving frame) and cosmic scale factor (as the internal evolution parameter). While for the description of evolution of the Dirac observables (i.e. the reduced theory) one invariant time parameter is sufficient. The evolution of the universe (as the dependence of the proper time on the internal evolution parameter) goes beyond the scope of the "equivalent" reduced theory and determines the measurable density of the matter field. "Particles" are the variables which diagonalize this measurable density. "Quasiparticles" are the variables which diagonalize the equations of motion and determine a set of integrals of motion. These definitions strongly differ from the conventional approach [8, 9, 10, 11] which goes from the conserved "particles" as initial data (i.e. constant coefficients of the field decomposition over classical solutions) to "unconserved quasiparticles" with diagonalization of the initial Hamiltonian, but not the equations of motion.

The Bogoliubov quasiparticles classify the states of the universe in both the classical theory and the quantum one. The states with a fixed number of quasiparticles are squeezed states 
for particles. The Bogoliubov quasiparticles allow us to define the state of "nothing" as the squeezed vacuum.

The formulation of classical and quantum versions of General Relativity in terms of measurable quantities leads to unification of classical and quantum cosmologies with QFT in GR and reveals the dependence of the classical evolution of the universe and the wave function of the universe on "methods of measurement and their standards".

An Einstein observer measuring lengths by the absolute standard sees the expansion of the universe, while a Weyl observer (who can measure the ratio of lengths of two vectors) sees the Hoyle-Narlikar evolution.

We considered the difference between a Weyl observer and an Einstein observer using, as an example, the evolution of the early universe. A Weyl observer (with relative standard of length) sees the squeezed vacuum inflation of particle masses due to the zero energy excitation

in the spectrum of quasigravitons, while an Einstein observer sees the linear dependence of the scale factor on the world time for the same dynamics.

\section{Acknowledgments}

We are happy to acknowledge interesting and critical discussions with B.M. Barbashov, P.N. Bogoliubov, A. Borowiec, A.V. Efremov, G.A. Gogilidze, V.G. Kadyshevsky, A.M. Khvedelidze, E.A. Kuraev, D. Mladenov, Yu.G. Palii, V.V. Papoyan, M. Pawlowski, and G.M. Vereshkov. One of the authors (V.P.) thanks A. Ashtekar, C. Isham, T. Kibble, J. Lukierski, L. Lusanna, and C. Rovelli for useful discussions.

\section{References}

[1] P.A.M. Dirac, "Lectures on Quantum Mechanics" (Belfer Graduate School of Science Yeshiva University, New York, 1964).

[2] L.D. Faddeev, A.A. Slavnov, Gauge fields: Introduction to Quantum Theory Benjamin-Gummings, 1984.

[3] M. Henneaux and C. Teitelboim 1992 Quantization of Gauge Systems (Princeton University Press, Princeton)

[4] A. Khvedelidze, Yu. Palii, V. Papoyan, V. Pervushin, Phys. Lett.B 402 p 263.

[5] L.N. Gyngazov, M.Pawlowski, V.N. Pervushin and V.I. Smirichinski Gen.Relativ.Gravit. 30 (1998) p 1749.

[6] M. Pawlowski, V. Papoyan, V. Pervushin, V. Smirichinski, Phys.Lett. B 444 (1998) p 293.

[7] J.C. Maxwell, A Treatise on Electricity and Magnetism Oxfrord, 1873.

[8] G.L. Parker, Phys.Rev. 183 (1969) p 1057;

Phys. Rev. D3 (1971) p 346.

[9] Ya.B. Zel'dovich, A.A. Starobinski, ZHETF 61 (1971) p 2161.

[10] L.P. Grishchuk, A.A. Sidorov, Phys.Rev. D42 (1990) p 3413. 
[11] A.A. Grib, S.G. Mamaev, V.M. Mostepanenko, Quantum effects in intensive external fields Moscow, Atomizdat, 1980 (in Russian).

[12] N. N. Bogoliubov, J.Phys. 11 (1947) p 23.

[13] P. A. M. Dirac, Proc.Roy.Soc. A 246 (1958) p 333;

P. A. M. Dirac, Phys. Rev. 114 (1959) p 924;

R. Arnovitt, S.Deser and C.W.Misner, Phys. Rev. 117 (1960) p 1595.

[14] A.L. Zelmanov, Dokl. AN USSR 107 (1956) p 315;

A.L. Zelmanov, Dokl. AN USSR 209 (1973) p 822;

Yu.S. Vladimirov, Frame of references in theory of gravitation, Moscow, Eneroizdat, 1982 (in Russian)

[15] V. N. Pervushin, V. I. Smirichinski, Phys.Atomic Nuclei 61 (1998) p 142; ibid 62 (1999) p 151.

[16] J.M Bardeen, Phys.Rev. D 22 (1980) p 1882;

H.Kodama, M.Sasaki, Prog. Theor. Phys. Suppl. 78 (1984) p 1.

[17] A. Lichnerowicz, Journ. Math. Pures and Appl. B 37 (1944) p 23.

[18] J. W. York. (Jr.), Phys. Rev. Lett. 26 (1971) p 1658;

K.Kuchar, J. Math. Phys. 13 (1972) p 768.

[19] R. Penrose, Relativity, Groups and Topology (Gordon and Breach, London, 1964);

N. A. Chernikov, E. A. Tagirov, Ann. Ins. Henri Poincare 9 (1968) p 109.

[20] H. Weyl, Berl.Ber. (1918) 465;

H. Weyl, Math.Zs. 2 (1918) p 384;

H. Weyl, Sitrungsber.d.Berl.Acad. (1918) p 465.

[21] J. V. Narlikar, Astrofizica e Cosmologia, Gravitazione, Quanti e Relativita ed. G. Barbera, (Firenze, 1979).

[22] Review of Particle Physics, Phys. Rev. D54 (1996) p 107. 